\title{
CONFORMALLY INVARIANT METRICS AND PRIME ENDS
}

\author{
CARL DAVID MINDA ${ }^{1}$
}

\begin{abstract}
Let $R$ be a Riemann surface such that the group of conformal self-mappings of $R$ acts transitively on $R$. If $d$ is a metric on $R$ which is invariant under all conformal automorphisms of $R$ and which induces the given topology on $R$, then it is shown that the metric space $\langle R, d\rangle$ is complete. This result is used to show that the prime end compactification of a simply connected Riemann surface $R$ cannot be obtained by completion of a metric space $\langle R, d\rangle$, where $d$ defines the given topology on $R$ and is conformally invariant.
\end{abstract}

Given a Riemann surface $R$, let $\mathscr{A}(R)$ denote the group of conformal self-mappings of $R$. $R$ is said to be conformally transitive if $\mathscr{A}(R)$ acts transitively on $R$; that is, if for every pair of points $p, q \in R$ there is an $f \in \mathscr{A}(R)$ with $f(p)=q$. A metric $d$ on $R$ is conformally invariant if, for all $p, q \in R$ and any $f \in \mathscr{A}(R), d(f(p), f(q))=d(p, q)$. The single result of this paper is to show that if $R$ is a conformally transitive Riemann surface and $d$ is a conformally invariant metric on $R$ which defines the given topology on $R$, then the metric space $\langle R, d\rangle$ is complete. This is applied to show that for a simply connected Riemann surface $R$ the prime end compactification of $R$ cannot be obtained as the completion of $\langle R, d\rangle$, where $d$ is a conformally invariant metric on $R$ compatible with the topology on $R$. Whether an analogous result holds for nonsimply connected Riemann surfaces is not known.

THEOREM. Let $R$ be a conformally transitive Riemann surface and $d$ be a conformally invariant metric on $R$ such that $d$ gives rise to the topology on $R$; then the metric space $\langle R, d\rangle$ is complete.

Proof. Let $\left(q_{n}\right)_{n=0}^{\infty}$ be a Cauchy sequence in the metric space $\langle R, d\rangle$; we wish to prove that this sequence converges to a point in $R$. Fix a

Received by the editors December 28, 1972 and, in revised form, July 20, 1973.

AMS (MOS) subject classifications (1970). Primary 30A72.

Key words and phrases. Conformal transitivity, conformally invariant metrics, prime ends.

${ }^{1}$ Research supported in part by National Science Foundation Grant GP-39051. 
point $p_{0} \in R$. Take $K$ to be a compact neighborhood of $p_{0}$ in the topology on $R$, then $K$ is also compact, and hence complete, in the metric space $\langle R, d\rangle$. Because $K$ is a neighborhood of $p_{0}$ in $\langle R, d\rangle$, there is an $\varepsilon>0$ such that $\left\{p \in R: d\left(p, p_{0}\right)<\varepsilon\right\} \subset K$. It is permissible to assume that $d\left(q_{m}, q_{n}\right)<\varepsilon$ for all $m$ and $n$. Since $\mathscr{A}(R)$ acts transitively on $R$, there is an $f \in \mathscr{A}(R)$ with $f\left(q_{0}\right)=p_{0}$. Set $p_{n}=f\left(q_{n}\right)$ for all $n$, then $\left(p_{n}\right)_{n=0}^{\infty}$ is also a Cauchy sequence in $\langle R, d\rangle$, because $d$ is conformally invariant, and $p_{n} \in K$ for all $n$. As $K$ is a complete subset of $\langle R, d\rangle$, there is a point $p \in K$ with $d\left(p_{n}, p\right) \rightarrow 0$. Then $d\left(q_{n}, q\right) \rightarrow 0$, where $q=f^{-1}(p)$, so $\langle R, d\rangle$ is complete.

In order to make use of this result, it is necessary to determine which Riemann surfaces are conformally transitive. Evidently, if $\mathscr{A}(R)$ acts transitively on $R$, then the group $\mathscr{A}(R)$ cannot be discontinuous. All Riemann surfaces $R$ for which $\mathscr{A}(R)$ is not discontinuous have been tabulated [7, pp. 243-244]; there are seven exceptional classes of Riemann surfaces. Among these exceptions the only ones with transitive conformal automorphism group are simply connected Riemann surfaces, compact Riemann surfaces of genus 1 and Riemann surfaces conformally equivalent to the twice punctured Riemann sphere.

The Theorem has an application to the theory of prime ends. Given a finitely connected Riemann surface $R$, let $R^{*}$ denote the Carathéodory prime end compactification of $R$ [3, pp. 358-367]. Mazurkiewicz [4], [5, pp. 261-269] obtained the prime end compactification of a bounded simply connected region $R$ in the complex plane by means of the completion of a metric space $\langle R, d\rangle$, where $d$ is the so-called prime end metric which is equivalent to the Euclidean metric on $R$. Later, Schlesinger [5, pp. 254-261], [6] defined prime ends for simply connected regions on the Riemann sphere in a conformally invariant manner through the use of extremal length. An elegant approach to the theory of prime ends could be obtained by a combination of these two approaches: define prime ends from the completion of a conformally invariant metric. This approach was taken by Gál [1], [2]. However, for simply connected Riemann surfaces such a program is doomed to failure since all metrics of the appropriate type are already complete. In particular, this shows that the prime end metric of Mazurkiewicz is not conformally invariant. Whether a similar result holds for Riemann surfaces which are not simply connected is not known.

\section{REFERENCES}

1. I. S. Gál, Conformally invariant metrics on Riemann surfaces, Proc. Nat. Acad. Sci. U.S.A. 45 (1959), 1629-1633. MR 22 \#3810.

2. - Conformally invariant metrics and uniform structures. I, II, Nederl. Akad. Wetensch. Proc. Ser. A $63=$ Indag. Math. 22 (1960), 218-231, 232-244. MR 22 \#9597. 
3. A. Hurwitz and R. Courant, Vorlesungen über allgemeine Funktionentheorie und elliptische Funktionen, Die Grundlehren der math. Wissenschaften, Band 3, Springer-Verlag, Berlin and New York, 1964. MR 30 \#3959.

4. S. Mazurkiewicz, Über die Definition der Primenden, Fund. Math. 26 (1936), 272-279.

5. M. Ohtsuka, Dirichlet problem, extremal length and prime ends, Van Nostrand Reinhold Math. Studies, no. 22, Van Nostrand Reinhold, New York, 1970.

6. E. C. Schlesinger, Conformal invariants and prime ends, Amer. J. Math. 80 (1958), 83-102. MR 20 \#1775.

7. G. Springer, Introduction to Riemann surfaces, Addison-Wesley, Reading, Mass., 1957. MR 19, 1169.

Department of Mathematics, University of Cincinnati, Cincinnati, Ohio 45221 